\title{
Ganglioneuroma en mediastino posterior en un paciente pediátrico: presentación de un caso clínico y revisión de la literatura
}

\author{
Carlos Guzmán-Valderrábano,凶 Lissa Antonieta de la Vega-Morales, \\ Raúl Hernández-Saldaña, Mario Soto-Ramos, Luis Carlos Hinojos-Gallardo
}

Hospital Infantil de Especialidades de Chihuahua, Universidad Autónoma de Chihuahua

Chihuahua, México.

Trabajo recibido: 22-VIII-2016; aceptado: 15-XII-2016

\begin{abstract}
RESUMEN. Los tumores del mediastino en la edad pediátrica son poco frecuentes y corresponden al 3\% del total de cirugías de tórax realizadas en este grupo de edad. Los tumores de origen neuroectodérmico son la principal causa de tumores de mediastino, siendo el ganglioneuroma el más benigno y menos frecuente con una incidencia de 1 en 100,000 niños. La mayor parte de estos tumores cursan asintomáticos y son diagnosticados de manera incidental; aunque, el 37\% secretan ácido mandélico (VMA) y/o ácido homovanílico (HVA) ocasionando síntomas como enrojecimiento, hipertensión o diarrea secretora. La resección quirúrgica por toracotomía o toracoscopia es el tratamiento de elección y su diagnóstico se realiza mediante el estudio histopatológico de la pieza quirúrgica. Por ser un tumor de escasa presencia, presentamos un caso clínico así como la revisión de la literatura.
\end{abstract}

Palabras clave: Ganglioneuroma, tumores neuroectodérmicos, neoplasias mediastínicas, cirugía, toracotomía.

ABSTRACT. Mediastinal tumors in children are rare and account for $3 \%$ of total chest surgeries performed in this age group. Neuroectodermal tumors are the leading cause of mediastinal tumors, being the ganglioneuroma the most benign and less frequent with an incidence of 1 in 100,000 children. Most of these tumors are asymptomatic and are diagnosed incidentally although $37 \%$ secrete mandelic acid (VMA) and/ or homovanillic acid (HVA) causing symptoms such as redness, hypertension or secretory diarrhea. The surgical resection by thoracotomy or thoracoscopy are the elective treatment and diagnosis is made by histopathology examination of the surgical specimen. As a rare tumor presentation, a clinical case report and a literature review is presented.

Key words: Ganglioneuroma, neuroectodermal tumors, mediastinal neoplasms, surgery, thoracotomy.

\section{PRESENTACIÓN DEL CASO}

Presentamos el caso de un paciente del género femenino de dos años nueve meses de edad sin antecedentes personales o familiares de importancia. Inició su padecimiento actual ocho meses previos al ingreso presentando evacuaciones disminuidas de consistencia de inicio súbito y progresando hasta ser de característica líquida, sin moco ni sangre, no fétidas, con un patrón habitual de dos a tres evacuaciones por día sin factores exacerbantes ni atenuantes, valorada y manejada en múltiples ocasiones sin presentar mejoría de los síntomas. Es referida a la consulta externa de pediatría por diagnóstico de diarrea crónica, donde a la exploración física no se encontraron datos patológicos específicos por lo que se le solicitan estudios paraclínicos y radio- grafía de abdomen observando una imagen anormal en tórax (figura 1).

Se realiza radiografía de tórax anteroposterior y lateral apreciando imagen radiopaca, homogénea, de bordes con apariencia bien limitados que no borra silueta cardíaca que compromete hemitórax izquierdo y mediastino. En proyección lateral se observa una imagen de las mismas características con bordes aparentemente bien limitados retrocardíaca corroborando el diagnóstico de tumoración en mediastino (figura 2). Se solicita tomografía computada de alta resolución de tórax simple y con contraste donde se observó en mediastino posterior y hasta región paravertebral izquierda una imagen sólida, ovoidea con bordes bien limitados con escaso reforzamiento en el borde posterior a la administración de contraste con diámetro mayor de 
$61 \mathrm{~mm}$. No se logró determinar si existía compromiso vertebral o medular (figura 3); por tanto, a través de una resonancia magnética de tórax con contraste pudo apreciarse una imagen hiperintensa homogénea con bordes bien limitados que realza tras la administración de medio de contraste descartándose compromiso en canal medular (figura 4).

Se solicitaron marcadores tumorales y catecolaminas en orina los cuales se reportaron negativos, el resto de los resultados de laboratorio estaban dentro de los percentiles para la edad. Con base en los resultados previos se realizó resección tumoral por toracotomía posterolateral izquierda, encontrando masa asintomática grande que se disecó a través de su cápsula logrando la disección completa y resección (figura 5). La paciente pasó a la Unidad de Cuidados Intensivos

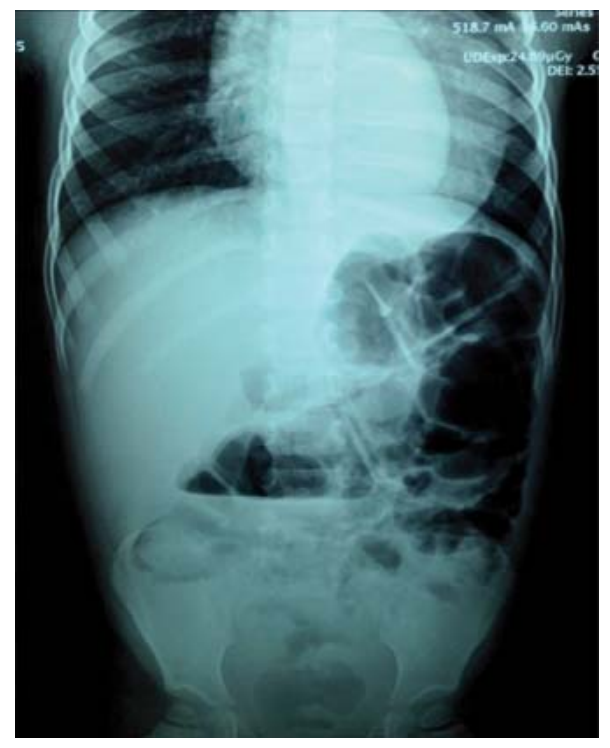

Figura 1. Radiografía de abdomen en decúbito. donde cursó con evolución favorable permaneciendo 24 horas en esta unidad, después cambió a la sala general observando mejoría en el patrón de evacuaciones de característica semisólida en número de uno a dos evacuaciones por día. Se retiró sello pleural 72 horas después de la cirugía y, por su favorable evolución, se decide el egreso continuando el seguimiento por consulta externa.

Se envía la pieza quirúrgica al Servicio de Anatomía Patológica quien reporta macroscópicamente un tumor mediastinal de $9.5 \times 7.5 \times 6 \mathrm{~cm}$ de diámetro, ovoideo con superficie externa lisa, cápsula íntegra café clara y blanda al corte con una superficie interna sólida, amarilla y blanda, sin evidencia de necrosis ni hemorragia. En la descripción microscópica refieren neoplasia constituida por estroma tipo células de Schwann, células fusiformes, sin datos de atipia ni pleomorfismo, con múltiples células ganglionares con núcleo grande, nucléolo prominente y abundante citoplasma eosinófilo, estableciendo el diagnóstico histopatológico de ganglioneuroma maduro de la clasificación patológica internacional de neuroblastomas (INPC) (figura 6).

\section{DISCUSIÓN}

Los tumores del mediastino en los niños son poco frecuentes, como grupo corresponden al $3 \%$ del total de cirugías de tórax realizadas en niños. Un tercio ocurre en menores de dos años de edad, manifestándose con mayor frecuencia en el mediastino posterior, como en el caso que se presenta. ${ }^{1}$ Del total de tumores del mediastino el $45-50 \%$ corresponden a neoplasias primarias, y de éstos entre el $40-45 \%$ a tumores malignos pudiendo fluctuar hasta un $72 \%{ }^{1}$

Se reportan como causa principal los tumores de origen neurogénico, ${ }^{2}$ constituyendo el $40-50 \%,{ }^{3}$ pueden derivar de las células ganglionares, paraganglionares
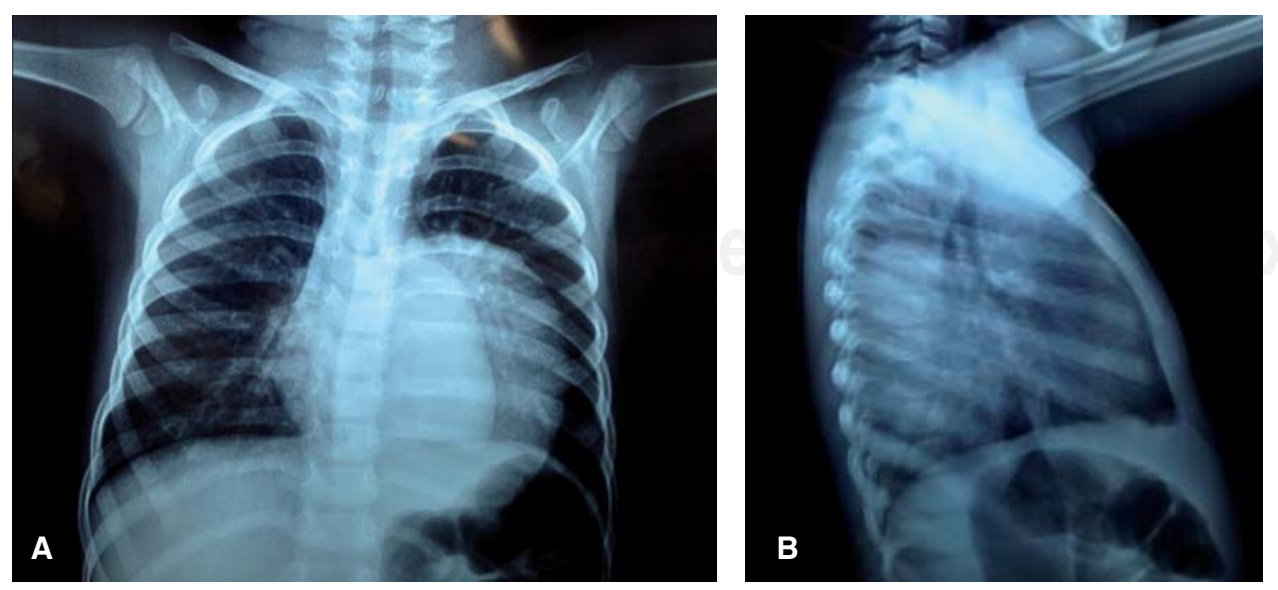

Figura 2.

Radiografías de tórax. Panel A) Proyección posteroanterior, se observa imagen radiopaca, homogénea de bordes bien limitados que no borra silueta cardíaca que compromete hemitórax izquierdo y mediastino. Panel B) Proyección lateral, se observa imagen radiopaca, homogénea con bordes bien limitados retrocardíaca. 

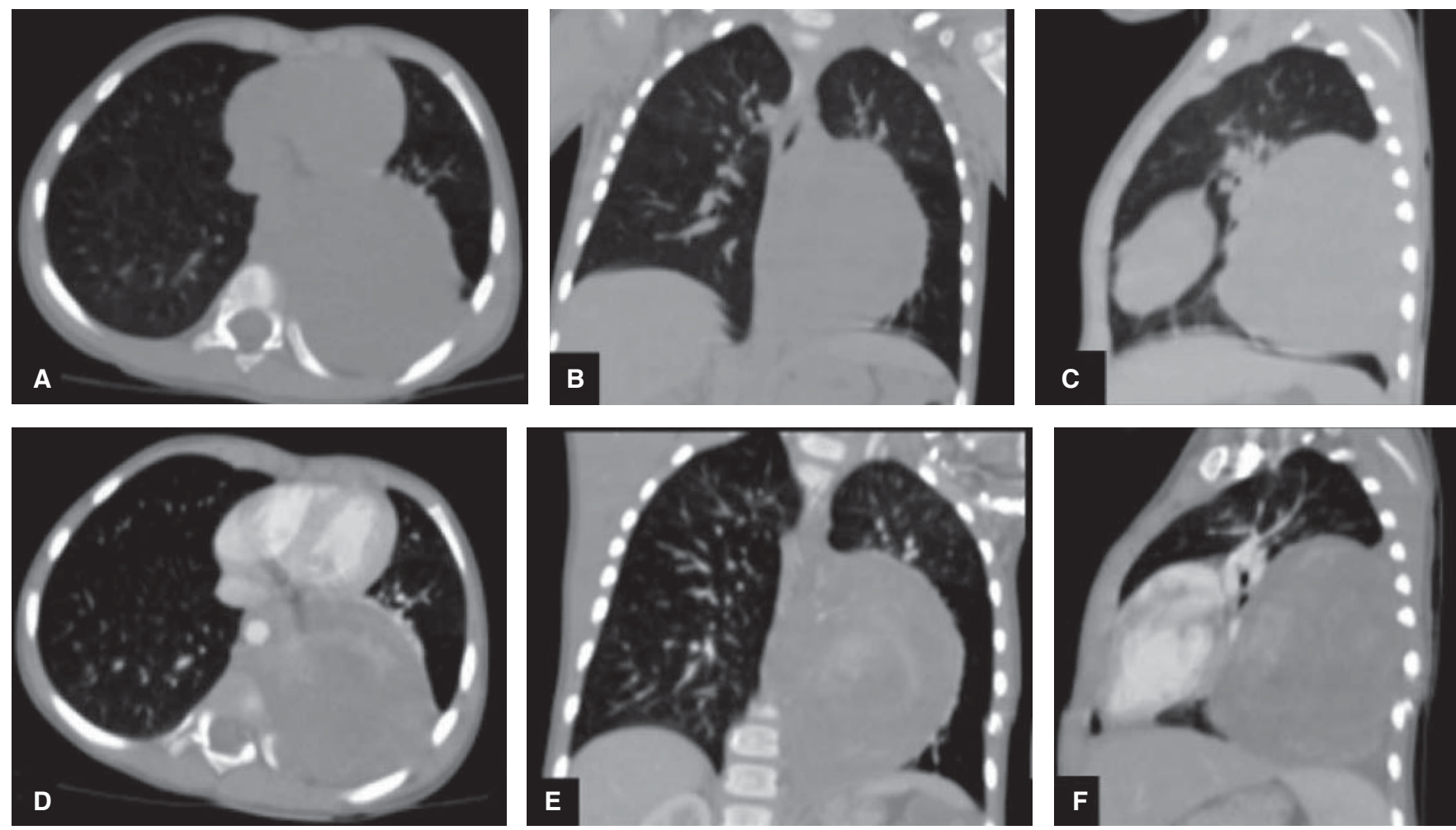

Figura 3. Tomografía de tórax de alta resolución. Se observa imagen sólida, ovoidea con bordes bien limitados en mediastino posterior con escaso reforzamiento en el borde posterior a la administración de contraste. Panel A) Tomografía simple, vista en proyección axial. Panel B) Tomografía simple, vista en proyección coronal. Panel C) Tomografía simple, vista en proyección sagital. Panel D) Tomografía en fase con contraste, vista en proyección axial. Panel E) Tomografía en fase con contraste, vista en proyección coronal. Panel F) Tomografía en fase con contraste, vista en proyección sagital.
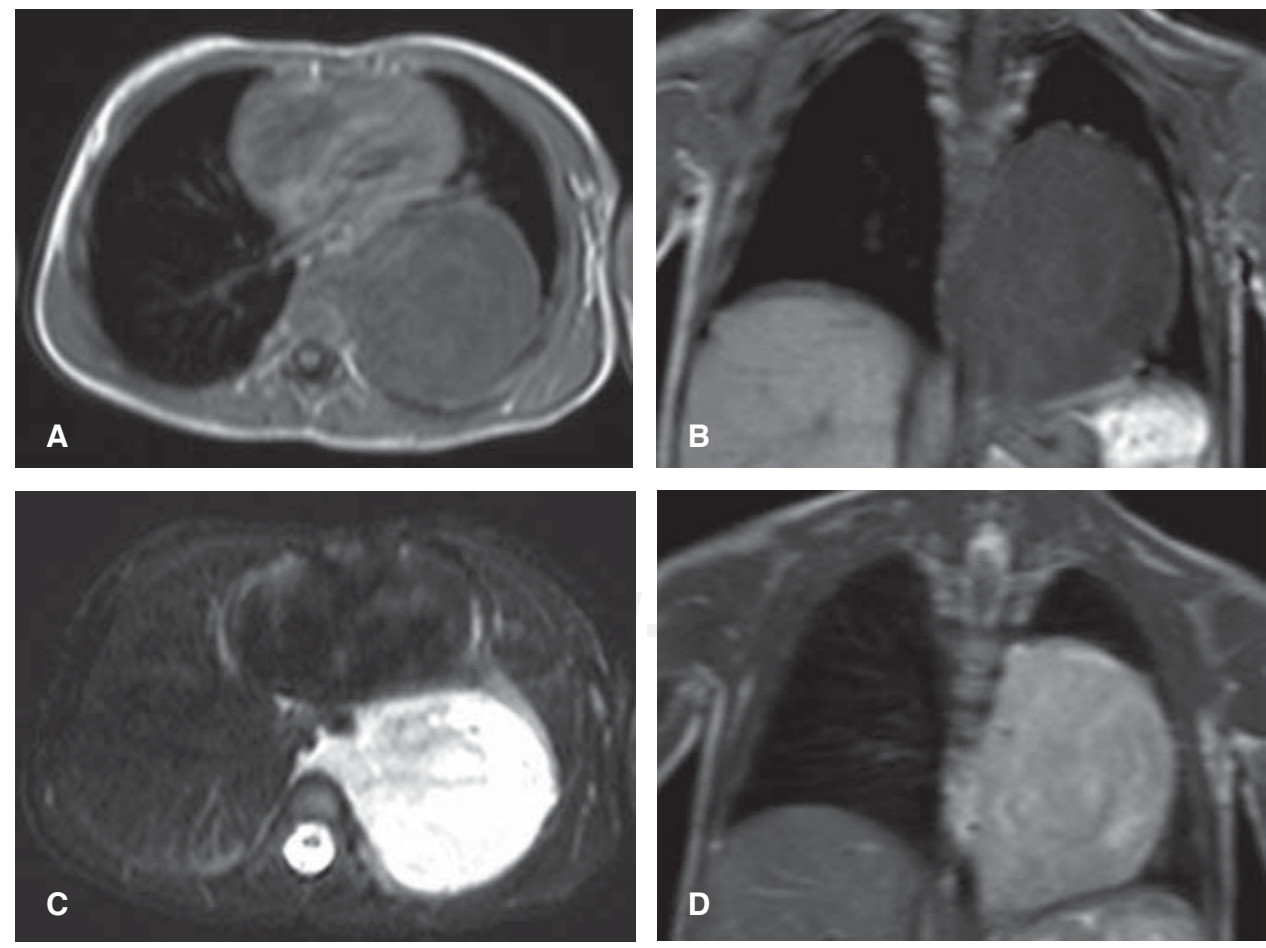

\section{Figura 4.}

Resonancia magnética de tórax. Se observa imagen hiperintensa homogénea con bordes bien limitados que realza tras la administración de medio de contraste sin encontrarse en relación con canal medular. Panel A) Vista en proyección axial. Panel B) Vista en proyección coronal. Panel C) Fase con contraste, vista en proyección axial. Panel D) Fase con contraste, vista en proyección coronal. 

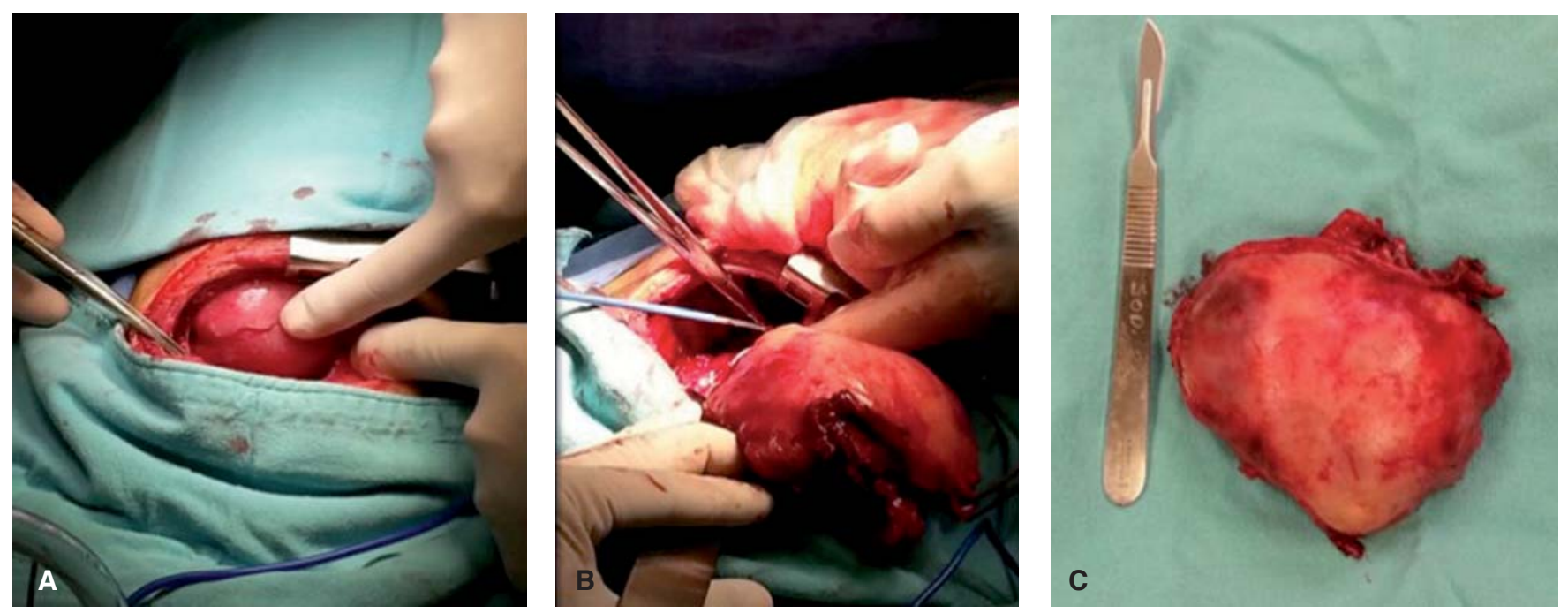

Figura 5. Toracotomía posterolateral izquierda. Panel A) Se observa masa mediastinal grande con cápsula lisa. Panel B) Disección y resección completa de masa mediastinal. Panel C) Pieza quirúrgica de masa tumoral de mediastino posterior.
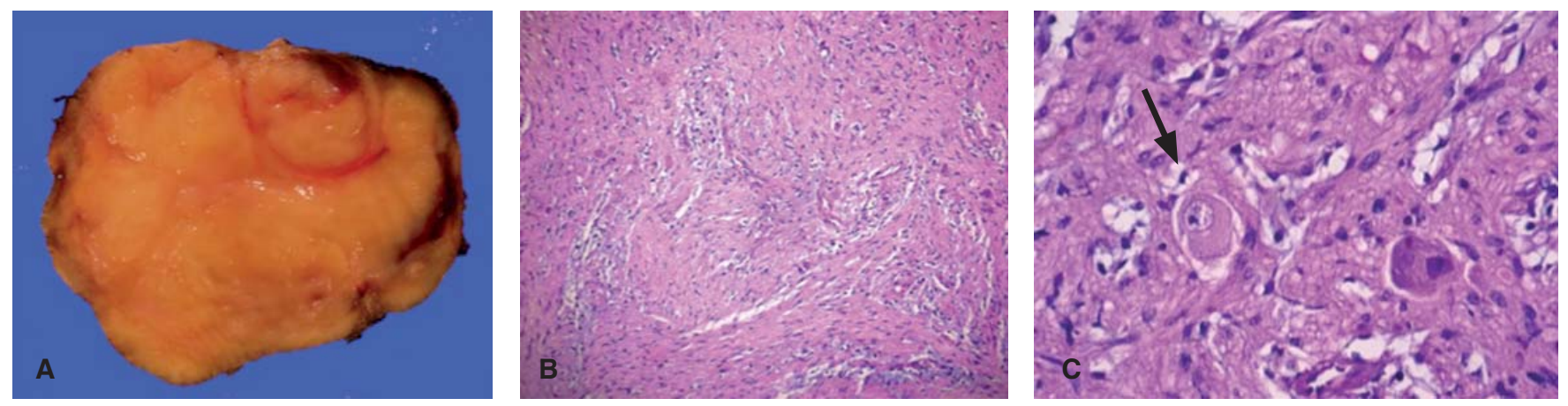

Figura 6. Imágenes histopatológicas. Panel A) Vista macroscópica. Al corte se observa una superficie homogénea, amarilla y blanda. Sin evidencia de necrosis, ni hemorragia. Panel B) Imagen en 10x. Se observa un fondo estromal constituido por células de Schwann en orientación longitudinal y transversal, con presencia de células ganglionares maduras dispersas entre el estroma de células de Schwann. Panel C) Imagen en 40x. Acercamiento en el cual se observa el detalle de una célula ganglionar madura con núcleo grande con cromatina abierta y nucléolo prominente con abundante citoplasma eosinófilo.

o del sistema nervioso simpático. El ganglioneuroma, ganglioneuroblastoma y el neuroblastoma son tumores que derivan de la cresta neural, difiriendo en su grado de maduración celular. ${ }^{4,5}$ El neuroblastoma es el más inmaduro, el más frecuente y el más agresivo; y el ganglioneuroma el más maduro, el más infrecuente $y$ benigno. ${ }^{5}$

El ganglioneuroma, como el presentado en este caso, es un tumor poco frecuente con una incidencia reportada de 1 en 100,000 niños; 6 ocurre predominantemente en la infancia ${ }^{7}$ con una media de presentación a los siete años. ${ }^{8}$ Se trata de un tumor benigno que se origina a partir de células de la cresta neural que componen los ganglios simpáticos a lo largo de la columna vertebral y glándulas suprarrenales. Comparten un linaje común histológico con el ganglioneuroblastoma y el neuroblastoma representando la forma diferenciada de este grupo, siendo el representante de los tumores periféricos neuroblásticos benignos. ${ }^{9}$ Los criterios del INPC (del inglés International Neuroblastoma Pathology Committee), definen al ganglioneuroma como un tumor de tejido dominante de estroma de Schwann, compuesto predominantemente por estroma ganglioneuromatoso con un menor componente de colecciones dispersas de neuroblastos diferenciados y/o células ganglionares maduras o en maduración, así como fue reportado en el estudio histopatológico del caso presentado.?

Puede surgir como un tumor primario o como una diferenciación espontánea inducida por el tratamiento de neuroblastoma o ganglioneuroblastomas. ${ }^{3}$ 
Con respecto al sitio de presentación del tumor, el caso clínico que se presenta corresponde con lo referido en la literatura, pues un $43 \%$ de estos casos son de manifestación intratorácica y por lo común localizados en los ganglios simpáticos del mediastino posterior. ${ }^{8}$ Sin embargo, también puede surgir en el retroperitoneo (37.5\%), glándula suprarrenal $(21 \%)$ y tejidos blandos retrofaríngeos (8\%). ${ }^{5,9}$

Clínicamente se manifiesta como una masa asintomática, la cual se descubre en un estudio radiográfico de tórax de rutina. A veces provoca un efecto de masa local ${ }^{10}$ pudiendo presentarse con síntomas derivados de la compresión del árbol traqueobronquial ${ }^{9} \mathrm{o}$ disnea en casos raros.

Los ganglioneuromas secretan VMA y/o HVA en un $37 \%$ de los casos, ${ }^{10}$ pudiendo manifestar enrojecimiento o hipertensión. También se han asociado con un síndrome de diarrea crónica, esta asociación se conoce como síndrome de Verner-Morrison o síndrome de WDHA (diarrea acuosa, hipokalemia y aclorhidria), el cual es causado por elevación del péptido intestinal vasoactivo, (VIP, vasoactive intestinal peptide, por sus siglas en inglés). Tomando en cuenta lo ya mencionado, la paciente cursó sin síntomas derivados del efecto de compresión sobre la vía respiratoria. Clínicamente cursó con diarrea crónica misma que se resolvió posterior a la resección quirúrgica del tumor que puede haber sido relacionado por efecto de la producción de catecolaminas; aunque no se documentó la elevación de las mismas en orina ni alteraciones electrolíticas.

En cuanto a los estudios de imagen, nuestro caso también corresponde radiológicamente a lo reportado en la literatura, la cual nos refiere que en la tomografía de tórax se comportan como masas sólidas, bien definidas, con comportamiento radiológico dependiendo de su componente de mayor proporción; y en resonancia magnética se observan hipointensos en $\mathrm{T} 1$ e hiperintensos en T2, realzando de forma variable tras la administración de gadolinio. Tanto la resonancia magnética como la tomografía computarizada aportan ventajas para la localización, tamaño y extensión del tumor, resultando ser de gran beneficio para el manejo quirúrgico. ${ }^{5,11}$

El diagnóstico depende del estudio patológico del tejido, los estudios biológicos preoperatorios y usando como apoyo los estudios de imagen. ${ }^{3}$ La resección quirúrgica es el tratamiento de elección. 2,7 Tanto la toracoscopia como la toracotomía han sido utilizadas para la resección de tumores del mediastino posterior. Con base en lo previo se decidió en la paciente, la resección quirúrgica por toracotomía logrando resecar la totalidad del tumor. ${ }^{3}$

Los ganglioneuromas son tumores de crecimiento lento y evolución benigna, ${ }^{5}$ el pronóstico es bueno y la recurrencia posterior a la cirugía es excepcional. ${ }^{12} \mathrm{Se}$ reportan como factores de buen pronóstico la aparición posterior a los cinco años de edad y la localización en el mediastino posterior. ${ }^{8}$

\section{REFERENCIAS}

1. Otárola D, Quinteros N, Palavecino T, Rostión C. Tumores del mediastino en niños. Revista Pediatría Electrónica 2009;6(2):41-48.

2. King RM, Telander RL, Smithson WA, Banks PM, Han MT. Primary mediastinal tumors in children. J Pediatr Surg 1982;17(5):512-520.

3. Lin PC, Lin SH, Chou SH, et al. Ganglioneuroma of posterior mediastinum in a 6-year-old girl: imaging for pediatric intrathoracic incidentaloma. Kaohsiung J Med Sci 2010;26(9):496-501. doi: 10.1016/S1607$551 \times(10) 70078-4$.

4. Akyildz EU, Yalcinkaya U. Thoracic neurogenic tumors: a clinicopathologic evaluation of 42 cases. Neurology Asia 2015;20(1):59-63.

5. Alcazar IM, Domínguez PA, González MR, Aguilar GJ. Caso clínico-radiológico. Rev Chil Pediatr 2010;81(5):461464.

6. Hopkins H, Gordils A, López J, Mariño C, Amundaray G. Ganglioneuroma: a propósito de un caso. Centro Médico 2004;49(2):94-99.

7. De Bernardi B, Gambini C, Haupt R, et al. Retrospective study of childhood ganglioneuroma. J Clin Oncol 2008;26(10):1710-1716. doi: 10.1200/JCO.2006.08.8799.

8. Agarwal KK, Sharma P, Kc SS, Bal C, Kumar R. Giant thoracic ganglioneuroma in a pediatric patient: staging and restaging with ${ }^{18} \mathrm{~F}-\mathrm{FDG}$ PET-CT. Rev Esp Med Nucl Imagen Mol 2014;33(5):310-311. doi: 10.1016/j. remn.2013.10.005.

9. Forsythe A, Volpe J, Muller R. Posterior mediastinal ganglioneuroma. Radiographics 2004;24(2):594-597.

10. Geoerger B, Hero B, Harms D, Grebe J, Scheidhauer K, Berthold F. Metabolic activity and clinical features of primary ganglioneuromas. Cancer 2001;91(10):19051913.

11. Guan YB, Zhang WD, Zeng QS, Chen GQ, He JX. CT and $M R I$ findings of thoracic ganglioneuroma. $\mathrm{Br} J$ Radiol 2012;85(1016):e365-e372. doi: 10.1259/bjr/53395088.

12. Alqoaer KI, Albalawi M, Alnoaiji MS, Khan A. Case report: Mediastinal ganglioneuroma presented as chronic diarrhea in a young Saudi girl. Int J Clin Med 2014;5:420-424.

\section{$\triangle$ Correspondencia:}

Dr. Carlos Guzmán-Valderrábano

Hospital Infantil de Especialidades de Chihuahua,

Universidad Autónoma de Chihuahua,

Chihuahua, México.

Correo electrónico: dr_valderrabano@hotmail.com drcarlosv@pediahero.com.mx

Los autores declaran no tener conflicto de intereses. 